\title{
Characterization of infrared dark clouds
}

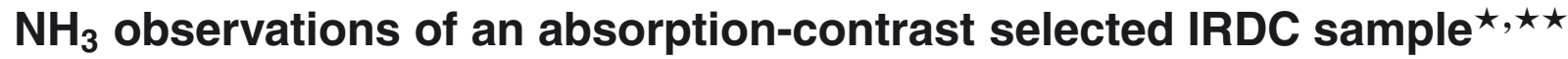

\author{
R.-A. Chira ${ }^{1}$, H. Beuther ${ }^{1}$, H. Linz ${ }^{1}$, F. Schuller ${ }^{2}$, C. M. Walmsley ${ }^{3,4}$, K. M. Menten ${ }^{5}$, and L. Bronfman ${ }^{6}$ \\ ${ }^{1}$ Max-Planck Institute for Astronomy, MPIA, Königstuhl 17, 69117 Heidelberg, Germany \\ e-mail: rox.chira@gmail.com \\ 2 European Southern Observatory, Alonso de Cordova 3107, Casilla 19001, Santiago 19, Chile \\ 3 Osservatorio Astrofisico di Arcetri, Largo E. Fermi 5, 50125 Firenze, Italy \\ ${ }^{4}$ Dublin Institute for Advanced Studies (DIAS), 31 Fitzwilliam Place, Dublin 2, Ireland \\ 5 Max-Planck-Institute for Radiostronomy, Auf dem Hügel 69, 53121 Bonn, Germany \\ ${ }^{6}$ Departamento de Astronomia, Universidad de Chile, Casilla 36-D, Santiago, Chile
}

Received 9 May 2012 / Accepted 18 December 2012

\section{ABSTRACT}

\begin{abstract}
Context. Despite increasing research in massive star formation, little is known about its earliest stages. Infrared dark clouds (IRDCs) are cold, dense and massive enough to harbour the sites of future high-mass star formation. But up to now, mainly small samples have been observed and analysed.

Aims. To understand the physical conditions during the early stages of high-mass star formation, it is necessary to learn more about the physical conditions and stability in relatively unevolved IRDCs. Thus, for characterising IRDCs studies of large samples are needed. Methods. We investigate a complete sample of 218 northern hemisphere high-contrast IRDCs using the ammonia (1,1)- and (2,2)-inversion transitions.

Results. We detected ammonia (1,1)-inversion transition lines in 109 of our IRDC candidates. Using the data we were able to study the physical conditions within the star-forming regions statistically. We compared them with the conditions in more evolved regions which have been observed in the same fashion as our sample sources. Our results show that IRDCs have, on average, rotation temperatures of $15 \mathrm{~K}$, are turbulent (with line width FWHMs around $2 \mathrm{~km} \mathrm{~s}^{-1}$ ), have ammonia column densities on the order of $10^{14} \mathrm{~cm}^{-2}$ and molecular hydrogen column densities on the order of $10^{22} \mathrm{~cm}^{-2}$. Their virial masses are between 100 and a few $1000 M_{\odot}$. The comparison of bulk kinetic and potential energies indicate that the sources are close to virial equilibrium.

Conclusions. IRDCs are on average cooler and less turbulent than a comparison sample of high-mass protostellar objects, and have lower ammonia column densities. Virial parameters indicate that the majority of IRDCs are currently stable, but are expected to collapse in the future.
\end{abstract}

Key words. stars: formation - ISM: clouds - ISM: molecules - ISM: abundances

\section{Introduction}

Despite their relatively small numbers, massive stars are important components of galaxies. However, we know little about the initial conditions for the formation of high-mass stars due to the difficulty of identifying the sites at which they form. Early stages of massive star formation should contain cold gas and dust cores with a peak in the spectral energy distribution around $200 \mu \mathrm{m}$ and no emission at near- and mid-infrared wavelengths. Using near- to mid-infrared Galactic plane surveys from the Midcource Space Experiment (MSX), the Infrared Space Observatory (ISO) and the Spitzer Space Observatory, it is possible to identify large samples of infrared dark clouds (IRDCs). These are dark, compact silhouettes seen against strong Galactic background emission (Simon et al. 2006; Peretto \& Fuller 2009; Perault et al. 1996; Egan et al. 1998). IRDCs have high molecular hydrogen column densities $\left(\sim 10^{22} \mathrm{~cm}^{-2}\right.$, Simon et al. 2006) and block background radiation, in particular at optical and near-infrared

\footnotetext{
$\star$ Appendices A and B are available in electronic form at http: //www . aanda. org

$\star \star$ Tables C.1-C.4 are only available at the CDS via anonymous ftp to cdsarc.u-strasbg.fr (130.79.128.5) or via http://cdsarc.u-strasbg.fr/viz-bin/qcat?J/A+A/552/A40
}

wavelengths. They cover broad ranges of gas masses from low-mass to high-mass star-forming regions. The IRDCs on the upper end of gas mass range are considered to be capable of harbouring high-mass star-forming regions in their earliest evolutionary stages. Based on the MSX database, Simon et al. (2006) built up a catalogue of about 10000 candidate IRDCs which are excellent starting points for studies of the early stages of high-mass star formation.

Since this catalogue is only based on mid-infrared absorption images, it lacks any additional information about the physical conditions within these regions. Therefore, further studies are needed. In this work we investigate the temperatures, turbulent properties and the virial parameters of IRDCs using the ammonia $\left(\mathrm{NH}_{3}\right)(J, K)=(1,1)$ - and $(2,2)$-inversion transitions. Ammonia has critical densities on the order of $10^{3} \mathrm{~cm}^{-3}$ and so its radio wavelength inversion transitions are good higherdensity tracers which suffer from minimal freeze out (Bergin \& Tafalla 2007). The (1,1)- and (2,2)-inversion lines can be observed simultaneously making ammonia also a good thermometer (Walmsley \& Ungerechts 1983). Since radiative transitions between different $K$-ladders are forbidden, metastable $(J, K)$ inversion level (with $J=K$ ) are only populated via collisions (Ho \& Townes 1983). From the observed ammonia spectra it is 
possible to determine a source's kinetic temperatures (Walmsley \& Ungerechts 1983), the level of turbulence via the line widths and its virial mass. Using the lines' rest velocities we can also calculate the kinematic distances of the sources using a model of the Galactic rotation curve (Reid et al. 2009).

Ammonia studies analysing star-forming gas clumps have also been conducted for less massive sources (e.g. Harju et al. 1991, 1993). It has been observed in high-mass regions for decades (Churchwell et al. 1990; Molinari et al. 1996; Sridharan et al. 2002; Schreyer et al. 1996), but most previous studies discussed smaller and/or more evolved samples (e.g., Sridharan et al. 2005; Pillai et al. 2006). The advantage of our sample is that it is large enough to study the physical properties of IRDCs statistically. Thus, we can compare our results to studies of objects in more evolved stages of high-mass star formation. In particular, we compare the physical conditions found in IRDCs with those in high-mass protostellar objects (HMPOs, by Sridharan et al. 2002). HMPOs comprise the next evolutionary stage where at the core centres massive protostars have formed already. Typical luminosities exceed are $10^{4} L_{\odot}$ and accretion processes are likely still ongoing (Sridharan et al. 2002).

Furthermore, the ammonia data will be compared with data on submm wavelength $(870 \mu \mathrm{m})$ dust emission collected in the course of the Atacama Pathfinder Experiment Telescope Large Area Survey of the Galaxy (ATLASGAL) survey (Schuller et al. 2009). With this additional data, we will be able to determine the sources gas masses, molecular hydrogen column densities and ammonia abundances and gain information about the stability of IRDCs.

Recently, Dunham et al. (2011) and Wienen et al. (2012) presented their works. They studied more heterogeneous samples (concerning the evolutionary stages of star formation in the observed regions) based on BOLOCAM and ATLASGAL (Rosolowsky et al. 2010; Schuller et al. 2009). Although they did not focus on IRDCs as we did, they have corresponding subsamples with sources at typical distances similar to ours. Thus, we will discuss similarities and differences between these studies and ours as well.

Further details about the selection criteria of our IRDC sample, the observations, data reduction and analysis are given in the Sects. 2-4. In Sect. 4, the IRDCs' rotation and kinetic temperatures, and column densities of ammonia are derived and compared with the data of the HMPO sample by Sridharan et al. (2002). Furthermore, we study the molecular hydrogen column density and ammonia abundance, as well as the stability of the IRDCs by analysing their virial parameters.

The paper is outlined as follows. Section 2 introduces details about our sample and the selection criteria. Section 3 provides information about the observations and data reduction. In Sect. 4 we present and discuss our results. In Sect. 5 we summarise them and state our conclusions.

\section{Source sample}

Based on the $8.3 \mu \mathrm{m}$ data collected by the MSX, Simon et al. (2006) compiled a catalogue of more than 10000 candidate IRDCs. Those contain about 12000 embedded Infrared Dark Cores that appear as high-contrast peaks within the IRDCs. We specifically selected Cores and refer to them here as IRDCs. In an attempt to present a complete sample of the northern hemisphere high-contrast IRDCs, our sample of 218 sources was selected from the catalogue of Simon et al. (2006) based on the following criteria.
Table 1. Number of sources being eliminated by the individual selection criteria.

\begin{tabular}{lc}
\hline \hline Selection criterion & Number of eliminated sources $^{a}$ \\
\hline (a) $\mathrm{NH}_{3}(1,1)$ line detected & 91 \\
(b) $8<T_{\text {rot }}<50 \mathrm{~K}$ & 17 \\
(c) $F_{870 \mu \mathrm{m}}^{40^{\prime \prime}}>4 \mathrm{rms}$ & 8 \\
\hline
\end{tabular}

Notes. ${ }^{(a)}$ Note that some sources have been eliminated, because they did not fulfil several of these criteria. Therefore, the sum of the listed sources here is bigger than the 109 sources which have been actually eliminated.

Firstly, the sources had to have contrast values greater than 0.3 , where contrast is defined as contrast $=$ (background image)/background (see Simon et al. 2006). This selects clouds with column densities of the order of $10^{22} \mathrm{~cm}^{-2}$ of molecular hydrogen or $10^{14} \mathrm{~cm}^{-2}$ of ammonia. For a source to be considered significant, the ratio of the peak-contrast versus its uncertainty has to be larger than three.

Secondly, the sources needed to be observable from Effelsberg for more than five hours and be larger than $0.6 \mathrm{arcmin}^{2}$ to fill the beam of $40^{\prime \prime} \mathrm{FWHM}$ at the given frequencies. Therefore, they have to be above an elevation of $20^{\circ}$ corresponding to Galactic longitudes roughly larger than $19^{\circ}$. Thus, our systematic analysis of our sample started at $l=19.27^{\circ}$ (it was also possible to observe nine sources with $l<19^{\circ}$ which are exceptions). The coordinates of the IRDCs we used, were taken from the catalogue based on the MSX observations (Simon et al. 2006). We see small differences compared to studies using (sub)mm peak positions. For consistency, we used the same positions for the ATLASGAL and Spitzer maps (cf. Sects. 4.5.2 and 4.5.4).

\section{Observation and data}

In 2008 and 2009, we used the Effelsberg $100 \mathrm{~m}$ telescope to observe simultaneously the $\mathrm{NH}_{3}(1,1)$ - and (2,2)-inversion lines with central rest frequencies of 23.6945 and $23.7226 \mathrm{GHz}$, respectively. The data were taken in a frequency switching mode with on average five minutes integration time per position. The weather was adequate with system temperatures mostly below $50 \mathrm{~K}$. The full width at half maximum (FWHM) is 40" and the velocity resolution is $\Delta v=0.38 \mathrm{~km} \mathrm{~s}^{-1}$. The absolute flux calibration was determined from observations of NGC 7027.

Fitting the whole hyperfine structure (HFS) of both lines using the standard procedures of CLASS (Hily-Blant et al. 2006), the brightness temperatures, $T_{\mathrm{mb}}$, local standard of rest velocities, $V_{\text {lsr }}$, line width FWHMs, $\Delta v$, and optical depths, $\tau$, were derived. The results are listed in Table C.1.

One has to consider that the HFS satellites, especially of the $(2,2)$ transition, have not been detected in every spectrum. This will be discussed in Sect. 4.1.1.

\section{Results}

\subsection{Distribution of detected IRDCs within the Galactic plane}

\subsubsection{Detection rate}

Out of our original sample of 218 sources 109 were detected in ammonia. The fit parameters of the sources with detected ammonia inversion transitions are listed in Tables C.1 to C.3. The 


\section{R.-A. Chira et al.: Characterization of infrared dark clouds}

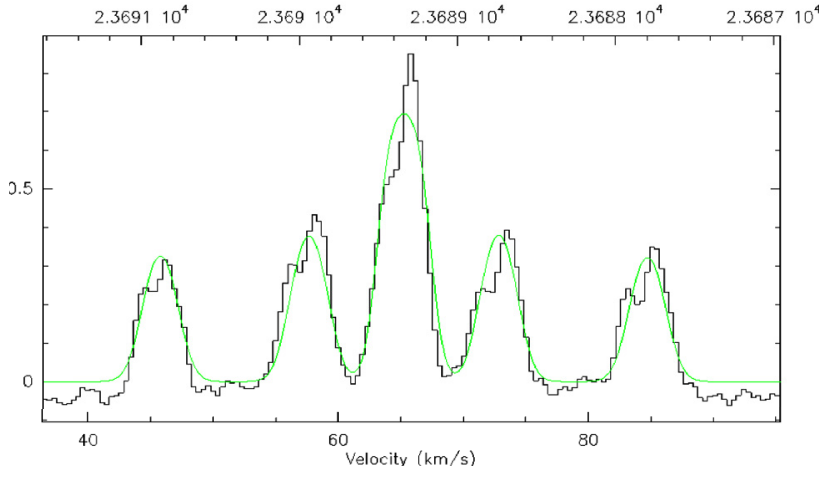

(a) (1,1)-inversion line

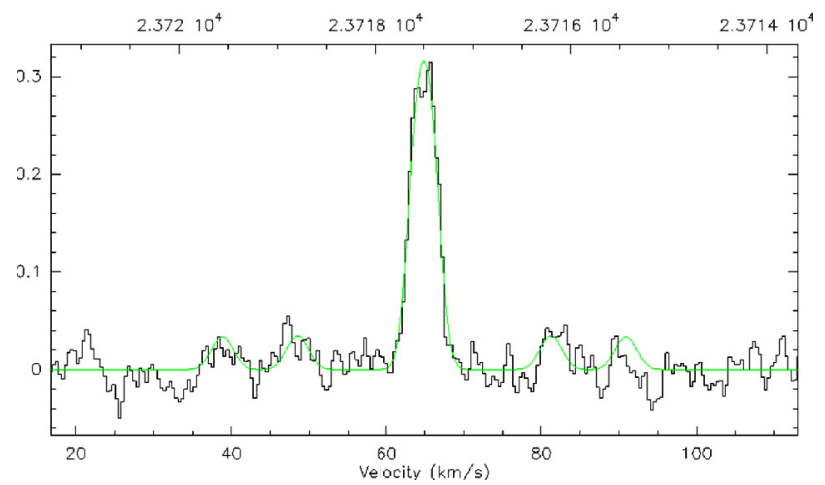

(b) (2,2)-inversion line

Fig. 1. Example for a good detection in ammonia. $\mathrm{NH}_{3}$ inversion lines in G19.92-00.29A. The green line indicates the hyperfine fit by CLASS.

formal errors $(1 \sigma)$ for the calculated quantities have been derived from Gaussian error propagation. IRDCs positions are indicated in Figs. B.1 and B.2. The black-and-white background is taken from the ATLASGAL dust emission maps by Schuller et al. (2009). The figures show a relatively good agreement between the compact sources detected by MSX extinction and by ATLASGAL.

For our analysis we applied the following criteria to obtain the significance of the statistics.

(a) The (1,1)-inversion line is detected and the brightness temperatures are higher than the threefold root-meansquare (rms).

(b) The rotation temperatures are between $8 \mathrm{~K}$ and $50 \mathrm{~K}$ and the absolute errors are lower than $20 \mathrm{~K}$. This is necessary, because both inversion lines are sensible temperature indicators, but only within this range (Danby et al. 1988).

(c) The flux densities $F_{v}^{40^{\prime \prime}}$ of the IRDCs' counterparts in the ATLASGAL dust emission maps are greater than four times the rms noise. This last criterion is needed for the virial analysis (cf. Sect. 4.5). In this case we were not able to derive independent gas masses or hydrogen column densities. If a source fulfils the first two criteria, but not the last one, its $\mathrm{NH}_{3}$ properties - rotation and kinetic temperatures, column densities of ammonia and the virial masses (Schilke 1989; Ho \& Townes 1983; MacLaren et al. 1988) - have been calculated, anyway, but not the virial parameter, hydrogen column density and ammonia abundance.

A good example for a detection fulfilling all of these criteria is G19.92-00.29A which is shown in Fig. 1. The green lines represent the fits to the HFS lines determined by CLASS.
81 out of 218 sources satisfied all our criteria. There were 29 sources without detectable $(2,2)$-inversion lines although the $(1,1)$-inversion lines was detected. In this case, it is not possible to derive exact parameters. But it is possible to give upper limits for the rotation temperature by using three-times the average RMS noise $(0.0925 \mathrm{~K}$, cf. Sect. A.1) as an upper limit for the (2,2)-line intensity (following Ho \& Townes 1983, cf. Sect. 4.2). In 91 sources the ammonia inversion transitions are not detected. For the sources not satisfying criterion (a) it is not possible to do any further calculations and analysis. There are 25 spectra with $(1,1)$-inversion lines lying within the noise. In this case the hyperfine fits by CLASS were not of good quality. A list of all sources toward which ammonia could not be detected is given in Table C.4.

17 sources were dropped, because their calculated rotation temperatures were not between 8 and $50 \mathrm{~K}$. This could be caused by poor signal-to-noise ratios and large errors in the hyperfine structure fits (cf. criterion (a)).

Eight sources turned out to have no significant counterparts in the ATLASGAL dust emission maps. As explained before we used the ammonia data to calculate temperatures, line widths and ammonia column densities for these sources and we list them in the tables in Appendix C.

Our sample and that of Wienen et al. (2012) have very little overlap. Depending on the search radii (defining the area around our sources where we searched corresponding sources of Wienen et al. 2012; in our case 20", 40", or 60") we find 10, 16 , or 22 sources in common. The properties derived by Wienen et al. (2012) match our results for the corresponding sources.

Our $\mathrm{NH}_{3}$ detection rate is $50 \%$. This lower than rates of $87 \%$ and $72 \%$ (respectively from Wienen et al. 2012 and Dunham et al. 2011) for samples which based on (sub)mm emission data. This indicates that sample selection solely based on mid-infrared absorption studies against the Galactic background are less reliable. Even if a contrast criterion is enforced, such studies can pick up a variety of structures that emulate IRDCs but might often turn out to be blown-out bubbles with very little dense gas inside (e.g. Wilcock et al. 2012). Comprehensive (sub)mm emission studies were not available at the time of our sample selection. The best future sample selections will be most likely based on a combination of mid-infrared absorption and (sub)mm emission selection criteria.

\subsubsection{Distribution within the Galactic plane}

Figure 2 shows the positions of the selected sources within the Galactic plane. The IRDCs lie close to the Galactic mid-plane (Galactic Latitude $b \sim 0^{\circ}$ ) where the gas density tend to be higher (Schuller et al. 2009; Rosolowsky et al. 2010; Beuther et al. 2012). As described in Beuther et al. (2012) in more detail the distribution along the Galactic latitude is symmetric and its peak is close to the Galactic mid-plane $\left(\sim 0.1^{\circ}\right)$. Therefore observations within a latitude range of $|b| \leq 0.5^{\circ}$ are recommended to reliably identify IRDCs.

In Fig. 3 the distributions of the sources with detected and non-detected ammonia $(1,1)$-inversion line have been plotted against the absolute value of their Galactic latitude b. For both distributions the mean values and medians have been calculated and are marked in Fig. 3. The IRDCs with detected inversion lines (mean: $(0.32 \pm 0.02)^{\circ}$, median: $\left.0.24^{\circ}\right)$ are significantly closer to the Galactic mid-plane than those without (mean: $(0.47 \pm 0.05)^{\circ}$, median: $\left.0.35^{\circ}\right)$. This implies that identification of IRDCs based on absorption studies against the Galactic background are less reliable at larger Galactic latitudes $b$. 


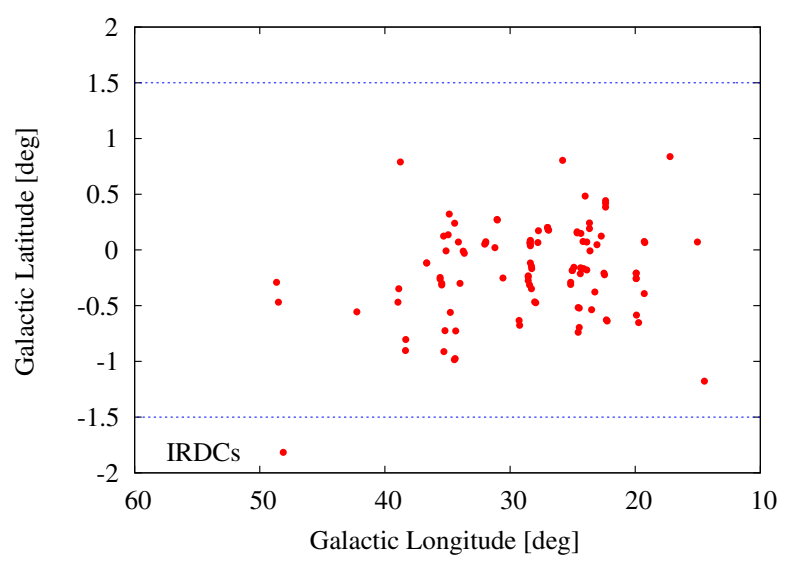

Fig. 2. Positions of selected IRDCs with detected ammonia transitions in the Galactic plane.

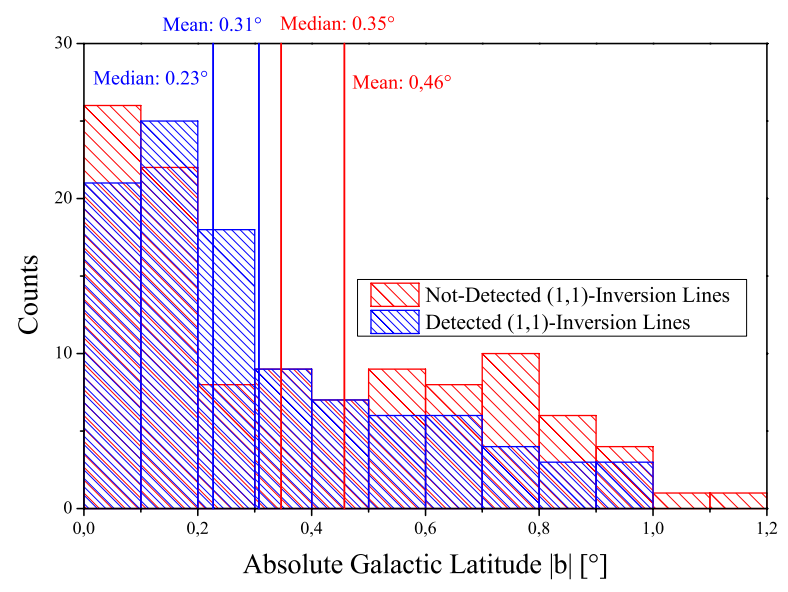

Fig. 3. Distribution of IRDCs with detected and without detected ammonia (1,1)-inversion line along the absolute Galactic latitude.

Schuller et al. (2009) and Beuther et al. (2012) have found a symmetric distribution of compact ATLASGAL sources around the mid-plane of the Milky Way. This is similar to our distribution of the sources where the ammonia $(1,1)$-inversion transition could be detected. But they have also detected a shift of the distribution peak to $b \sim-0.1^{\circ}$. However our sample is too small to do a similar analysis.

\subsubsection{Distance}

In this and the following sections, the results for the physical conditions and virial parameters will be presented and discussed. Therefore, we only take the 109 sources into account which were detected in the ammonia.

To be able to calculate the IRDCs' virial masses, which will be averaged over the beam, we needed the sources' radii. For estimating them, we derived the kinematic distances between the sources and the sun. Using a model for the Galactic rotation with new parameters proposed by Reid et al. (2009), (near and far) kinematic distances of a source with measured LSR velocity at given Galactic coordinates can be derived. The method is described in more details in the paper by Reid et al. (2009). Given that the sources in our sample appear in silhouette against background radiation, we chose the near values for sources with a distance ambiguity.

The IRDCs are at distances of a few kilo-parsecs (cf. Table 2 and Table C.2) and have radii between $0.25 \mathrm{pc}$ and $0.5 \mathrm{pc}$. To estimate these, we assumed the FWHM of the Effelsberg

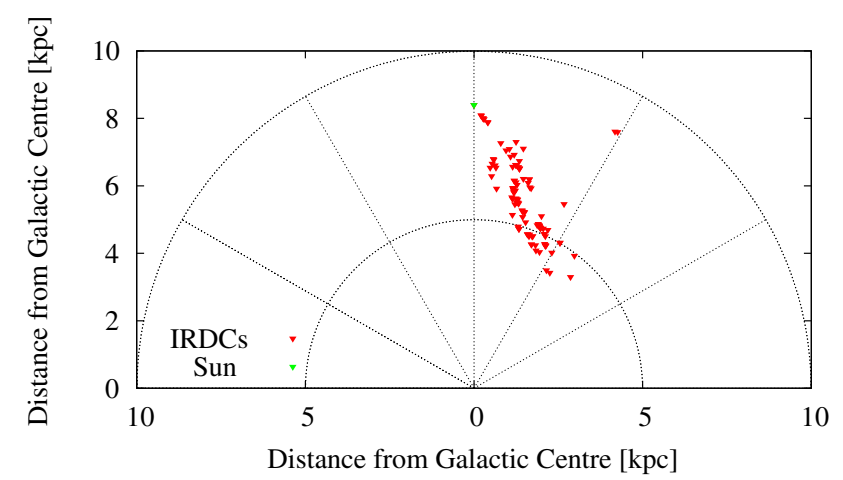

Fig. 4. Positions of selected IRDCs within the Galaxy.

Table 2. Statistics of IRDC sample.

\begin{tabular}{lcccc}
\hline \hline IRDC parameter $^{a}$ & Mean & Min. & Median & Max. \\
\hline$T_{\text {rot }}[\mathrm{K}]$ & 15 & 8 & 14 & 29 \\
$T_{\text {kin }}[\mathrm{K}]$ & 18 & 8 & 16 & 41 \\
$N_{\mathrm{NH}_{3}}\left[10^{14} \mathrm{~cm}^{-2}\right]$ & 7.0 & 0.9 & 5.1 & 32.0 \\
$N_{\mathrm{H}_{2}{ }^{\prime \prime}}^{1{ }^{\prime}}\left[10^{22} \mathrm{~cm}^{-2}\right]$ & 3.5 & 0.1 & 2.9 & 27.3 \\
$\chi_{\mathrm{NH}_{3}}\left[10^{-8}\right]$ & 3.8 & 0.6 & 1.9 & 45.9 \\
$\Delta v_{1}\left[\mathrm{~km} \mathrm{~s}^{-1}\right]$ & 1.7 & 0.7 & 1.6 & 4.0 \\
$d[\mathrm{kpc}]$ & 3.2 & 0.4 & 3.2 & 5.9 \\
\hline$M_{\mathrm{vir}}^{b}\left[M_{\odot}\right]$ & 325 & 6 & 264 & 1099 \\
$\alpha_{\mathrm{OH}}{ }^{c}$ & 6.1 & 0.3 & 2.3 & 155.2 \\
$\alpha_{\mathrm{MRN}}{ }^{4}$ & 1.9 & 0.1 & 0.7 & 49.4 \\
\hline
\end{tabular}

Notes. The statistics have been built up by the 109 IRDCs with detected ammonia transitions. ${ }^{(a)}$ Note that $T_{\text {rot }}$ represents the rotation temperature, $T_{\text {kin }}$ the kinetic temperature, $N_{\mathrm{NH}_{3}}$ the column density of ammonia, $N_{\mathrm{H}_{2}}^{19^{\prime \prime}}$ the column density of molecular hydrogen, $\chi_{\mathrm{NH}_{3}}$ the abundance of ammonia, $\Delta v_{1}$ the line width FWHM of the ammonia (1,1)-inversion line, $\mathrm{d}$ the distance between source and sun, $M_{\text {vir }}$ the virial mass and $\alpha_{\mathrm{i}}$ the virial parameters for the used models (cf. Sect. 4.5). ${ }^{(b)}$ The here given virial mass $M_{\text {vir }}$ is the mean value of the virial masses for the individual density profiles. Cf. Sect. 4.5.1 for more details. ${ }^{(c)}$ These are the sources' virial parameters, giving the ratio between kinetic and gravitational energy and, thus, an estimation for the stability of the sources. These Parameters will be discussed in more detail in Sect. 4.5.3.

beam $\left(40^{\prime \prime}\right)$ as a proxy for the size. Their positions within the Galactic plane and Galaxy are sketched in the Figs. 2 and 4.

\subsection{Rotation and kinetic temperature}

For deriving the rotation temperatures and column densities, we followed the outlines by Schilke (1989), Ho \& Townes (1983) and Ragan et al. (2011). For a better understanding we shortly summarise the most important points in Appendix A.

The calculated results for all sources are presented in Table C.2. The errors have been derived from Gaussian error propagation. The same applies to all other quantities if not mentioned otherwise.

The statistical properties of the sample are summarised in Table 2 including the mean values and the medians of the derived quantities, as well as the minimal and maximal values.

The IRDCs have rotation temperatures between $8 \mathrm{~K}$ and $29 \mathrm{~K}$. Although the latter being an upper limit, the majority of the IRDCs have mean rotation temperatures around $15 \mathrm{~K}$ (median: $14 \mathrm{~K}$ ) being more obvious in Fig. 5. It shows a cumulative distribution of our sample's rotation temperatures 


\section{R.-A. Chira et al.: Characterization of infrared dark clouds}

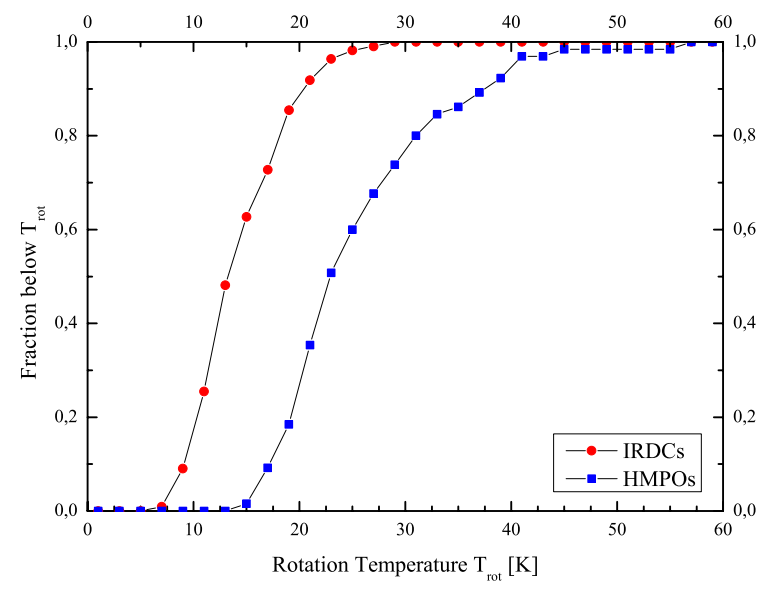

Fig. 5. Cumulative distributions of $\mathrm{NH}_{3}$ rotation temperatures of IRDCs (red data points) and HMPOs (blue data points).

(red data points). About $80 \%$ of the sources have values between 10 and $20 \mathrm{~K}$. This is consistent with previous studies (Pillai et al. 2006; Peretto et al. 2010; Ragan et al. 2011).

In addition, the blue points show the cumulative distribution of rotation temperatures derived for the sample of 65 HMPOs from Sridharan et al. (2002). This sample had been observed in the same fashion as ours with the Effelsberg telescope. Therefore, the results for rotation temperatures, line width FWHMs and column densities can be compared with the results for our sample IRDCs here. HMPOs are considered to be the next evolutionary step in high-mass star formation after IRDCs (Beuther et al. 2007).

The sample of HMPOs has a mean rotation temperature of $26 \mathrm{~K}$ (median: $24 \mathrm{~K}$ ) which is significantly higher than the value we find for our IRDCs. Therefore, the rotation temperature is a good indicator for differentiating between evolutionary stages: the rotation temperature increases with evolution. While HMPO temperatures and luminosities mainly stem from internal heating processes (hydrogen burning and accretion), the IRDC temperatures and luminosities are dominated by external radiation fields (Wilcock et al. 2012).

We derived kinetic temperatures with the approximation of Tafalla et al. (2004). More details are given in Appendix A.1. We estimate that they have errors of $5 \%$. The kinetic temperatures of the IRDCs vary between $8 \mathrm{~K}$ and $41 \mathrm{~K}$ with a mean value of $18 \mathrm{~K}$ and a median of $16 \mathrm{~K}$. These values are similar to those of the rotation temperature. This is not surprising, because both quantities are nearly linearly connected until $20 \mathrm{~K}$ and, thus, approximately equal within this regime. (Danby et al. 1988). Our results match those of Wienen et al. (2012, median $T_{\text {rot }}$ of $17 \mathrm{~K}$ ) and Dunham et al. (2011, mean $T_{\text {kin }}$ of $15.6 \mathrm{~K}$ ). Furthermore, the trend toward higher rotation temperatures and line widths (cf. Sect. 4.3) with progressing protostellar evolution agrees with the results of Wienen et al. (2012) who compared the relative number and cumulative distributions of different stages between IRDCs and the envelopes of ultracompact HII regions.

Comparing our results with those of Harju et al. (1991, 1993) (mean $T_{\text {kin }}$ between 14 and $20 \mathrm{~K}$ ), our sources have, on average, the same temperature or are slightly cooler. This can be explained by taking into account that Harju et al. included also sources being associated with IRAS sources heating up their envelopes.

Using Spitzer $24 \mu \mathrm{m}$ data we checked for associations with young stellar object (YSO) to identify a sample of starless IRDCs. We looked for any emission source within a circular area

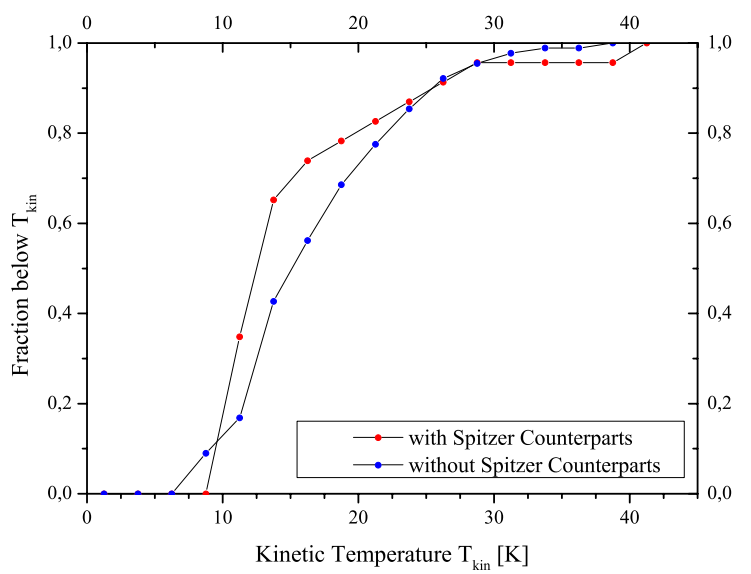

Fig. 6. Cumulative distributions based on the sources where $\mathrm{NH}_{3}$ has been detected. The red subsample includes the sources with Spitzer $24 \mu \mathrm{m}$ association, the blue subsample the ones without.

of radius $20^{\prime \prime}$ around the position. The results are listed in the last column of Table C.2. We found that 87 (79.8\%) IRDC candidates have no counterpart. But 22 (20.2\%) of our sample IRDCs are associated with YSOs or lie close enough to a Spitzer $24 \mu \mathrm{m}$ source that the beam includes parts of them. We also investigated whether there exists a correlation between the (non)detection in Spitzer $24 \mu \mathrm{m}$ and the kinetic temperature. However, no statistically significant difference between the two subsamples could be identify (cf. Fig. 6). This could be explained by considering our beam which covers a greater volume than the still too small and too young protostars are able to influence. While the line widths of sources with and without Spitzer counterparts are almost identical, there is a trend of higher $\mathrm{H}_{2}$ and $\mathrm{NH}_{3}$ column densities for sources with Spitzer counterparts. However, since the sample with Spitzer $24 \mu$ m detections is relatively small (22), it is unclear whether this is statistically significant.

\subsection{Line width}

Another observable to characterise IRDCs and HMPOs is the line width. The IRDCs have $\mathrm{NH}_{3}(1,1)$ line widths of around $1.7 \mathrm{~km} \mathrm{~s}^{-1}$ (mean and median) which agrees with the results of Wienen et al. (2012), but is higher than the values of Harju et al. (1991, 1993). The latter can be explained by taking into account that the sources studied by Harju et al. (1991) are at much smaller distances than ours. Thus, for the same beam one sees smaller size scales and hence smaller FWHMs which agrees with the Larson line width - size relation.

We investigated possible correlations between the FWHM and the $\mathrm{H}_{2}$ column density as well as between the line width and the kinetic temperature. However, no clear correlation between these quantities was found.

Figure 7 shows that the IRDCs are not only colder than the HMPOs, but have also narrower lines (for HMPOs: mean: $2.1 \mathrm{~km} \mathrm{~s}^{-1}$, median: $2.0 \mathrm{~km} \mathrm{~s}^{-1}$ ). The line widths are larger than expected for thermal emission at the given temperatures $\left(\approx 0.2 \mathrm{~km} \mathrm{~s}^{-1}\right.$ ) observed in low-mass star-forming regions (Kunth et al. 1996). The increased line widths can be caused by intrinsic cloud turbulence, infall motions or outflows from young forming stars. 


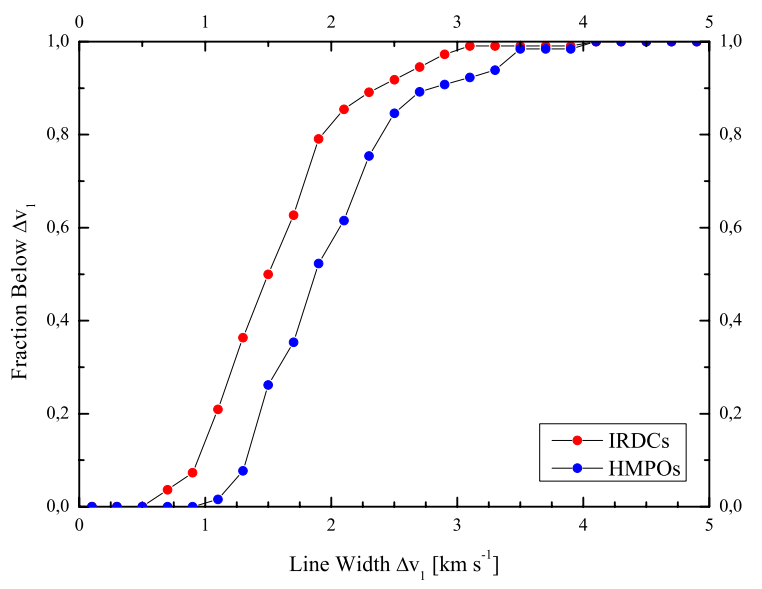

Fig. 7. Cumulative distributions of $\mathrm{NH}_{3}$ line width FWHMs of IRDCs (red data points) and HMPOs (blue data points).

\subsection{Ammonia column density}

Analogous to the temperatures the derivation of the column density can be found in Appendix A.2. The column densities for the IRDCs are listed up in Table C.2.

The ammonia column densities range between $9 \times 10^{13} \mathrm{~cm}^{-2}$ and $3 \times 10^{15} \mathrm{~cm}^{-2}$. The mean value is approximately $7 \times$ $10^{14} \mathrm{~cm}^{-2}$, the median is about $5 \times 10^{14} \mathrm{~cm}^{-2}$ (cf. Table 2). As the rotation temperature and the line width, also the column density of ammonia is an observable for the evolution of star-forming regions. Figure 8 shows that the column densities of our IRDCs are on average smaller than those of the HMPO sample (mean: $4 \times 10^{15} \mathrm{~cm}^{-2}$, median: $3 \times 10^{15} \mathrm{~cm}^{-2}$ ). This can be interpreted as evidence that the column densities of ammonia do change on spatial scales traced by single-dish telescopes in these early stages of massive star formation. The ammonia column densities of our sample are about one order of magnitude smaller than the ones of Wienen et al. (2012), but agree with the ones of Harju et al. (1991, 1993) and Dunham et al. (2011). Lower average column densities could be caused by our selection criteria based on absorption shadows. It appears that the extinction features trace the column density peaks less well than the ATLASGAL submm continuum observations.

\subsection{Hydrogen properties and virial parameter}

\subsubsection{Virial mass}

When we derived the virial mass $M_{\text {vir }}$, we only considered the balance between bulk kinetic and gravitational energy. Solving this for the mass and assuming a Gaussian velocity distribution, the virial mass can be calculated by

$M_{\mathrm{vir}}=k_{2} r \Delta v_{1}^{2}$

where $r$ represents the radius of the source and $\Delta v_{1}$ its FWHM in ammonia (1,1)-inversion transition (MacLaren et al. 1988). $k_{2}$ is a constant depending on the assumed density profile (see Table 3).

It is not clear whether the sources' density profiles follow a $r^{-1}$ - or $r^{-2}$-power-law. Therefore, the masses $M_{\mathrm{vir}}^{r^{-1}}$ and $M_{\mathrm{vir}}^{r^{-2}}$ were calculated with both power-laws. They are presented in Table C.2. For Table 2 and the further calculations of the virial parameters (cf. Sect. 4.5.3) we used the mean value $M_{\text {vir }}=$ $\frac{1}{2}\left(M_{\mathrm{vir}}^{r^{-1}}+M_{\mathrm{vir}}^{r^{-2}}\right)$.

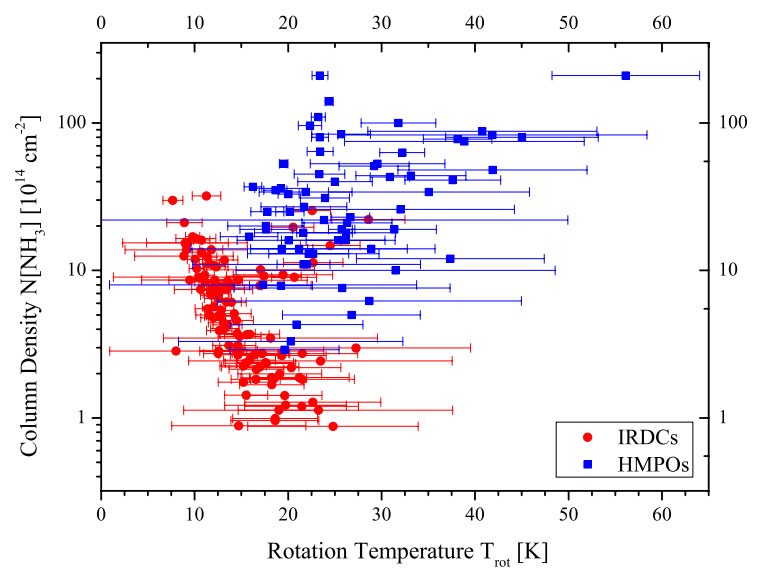

Fig. 8. Correlation plot between the $\mathrm{NH}_{3}$ total column density and the $\mathrm{NH}_{3}$ rotation temperature of the IRDCs (red data points) and the HMPOs (blue data points).

The (beam averaged) virial masses range between $6 M_{\odot}$ and $1100 M_{\odot}$ with a mean value of approximately $325 M_{\odot}$ and a median of $264 M_{\odot}$.

\subsubsection{Hydrogen gas mass}

For the calculation of the gas masses, we used the dust emission maps of the ATLASGAL project (Schuller et al. 2009), more precisely the sources' flux densities. For this part we made use of the Gildas GREG package (Buisson et al. 2007). Since the ATLASGAL maps have a HPBW of 19.2" we need to smooth them. Otherwise we would not be able to compare them with our Effelsberg data (40" HPBW). We did that by folding the ATLASGAL maps with a Gaussian of $\sqrt{\left(40^{\prime \prime}\right)^{2}-\left(19^{\prime \prime}\right)^{2}}=$ $35.1^{\prime \prime}$. Then, we measured the flux densities $F_{v}$ at the locations of the individual IRDC peaks. The errors are estimated to be $20 \%$ of the measured flux densities. This is sufficient, because the gas masses' errors are dominated by the error of the dust absorption coefficient. The gas mass $M_{\text {gas }}$ is given by

$M_{\mathrm{gas}}=\frac{d^{2} F_{v}^{40^{\prime \prime}} R}{B_{v}\left(T_{\mathrm{D}}\right) \kappa_{v}}$

where $d$ is the source's distance to the sun, $F_{v}^{40^{\prime \prime}}$ the total flux density measured in dust emission at $870 \mu \mathrm{m}(v=345 \mathrm{GHz})$ within the smoothed beam of $40^{\prime \prime} . B_{v}\left(T_{\mathrm{D}}\right)$ represents the Planck function for the dust temperature $T_{\mathrm{D}}$ being approximately equal to the kinetic temperature $T_{\text {kin }}$ for temperatures below $50 \mathrm{~K}$ in the dense environment of IRDCs, since gas and dust temperature are collisionally coupled at high densities in our IRDCs. Furthermore also the kinetic and the rotation temperatures are approximately equal below $50 \mathrm{~K}$. Thus, we inserted the rotation temperature $T_{\text {rot }}$ for the dust temperature.

$R$ represents the gas-to-dust mass ratio and $\kappa_{v}$ the dust absorption coefficient. Following Schuller et al. (2009), we assume $R$ to be 100 and $\kappa_{\mathrm{OH}}=1.85 \mathrm{~cm}^{2} \mathrm{~g}^{-1}$ (Schuller et al. 2009; Ossenkopf \& Henning 1994). But since these values are just estimated, they contain the biggest uncertainties, particularly the dust absorption coefficient.

In the paper by Ossenkopf \& Henning (1994), the dust absorption coefficient dependency on different conditions is described in more detail and the older data of Mathis et al. (1977) are compared with newly derived values. Ossenkopf \& Henning list the dust absorption coefficient in dependency of the wavelength and used gas density. The dust absorption coefficient 
Table 3. Virial theorem coefficient (MacLaren et al. 1988).

\begin{tabular}{lc}
\hline \hline Density distribution & $k_{2}$ \\
\hline$\rho \sim r^{-1}$ & 190 \\
$\rho \sim r^{-2}$ & 126 \\
\hline
\end{tabular}

of $\kappa_{\mathrm{OH}}=1.85 \mathrm{~cm}^{2} \mathrm{~g}^{-1}$ was interpolated from Table 1, Col. 5 (Ossenkopf \& Henning 1994; Schuller et al. 2009) at a wavelength of $870 \mu \mathrm{m}$. By interpolating the original data of Mathis et al. - given in Table 1, Col. 2 (Ossenkopf \& Henning 1994) - we determined the dust absorption coefficient to be $\kappa_{\mathrm{MRN}}=$ $(0.6 \pm 0.1) \mathrm{cm}^{2} \mathrm{~g}^{-1}$. Comparing this with $\kappa_{\mathrm{OH}}$, this implies an error factor of about 3 in gas mass. Since the exact value is not known, the two models give us an estimate of the upper and lower limits of gas masses (cf. Table 2). We calculated the gas masses for all sample sources with both models, being labelled as $M_{\mathrm{OH}}$ and $M_{\mathrm{MRN}}$ (as being the corresponding virial parameters).

Nevertheless, our sources with masses up to several thousand solar masses are on average more massive than the clumps of Harju et al. (1991, 1993). This is not surprising, because our sample was selected for high-mass cores, while Harju et al. (1991, 1993) observed mainly low- to intermediate-mass cores.

\subsubsection{Virial parameter}

The virial parameter for molecular clouds is defined by

$\alpha=a \frac{2 T}{|W|}=\frac{M_{\mathrm{vir}}}{M_{\mathrm{gas}}}$

(Bertoldi \& McKee 1992) where the dimensionless parameter $a$ is the correction factor for sources having no uniform and spherical mass distribution. $T$ and $W$ represent the kinetic and gravitational energies. Their ratio is equivalent to the ratio of virial and gas mass.

The virial parameter is a helpful tool, because it is an indicator for a source being in virial equilibrium or not. In a simplified picture, a source is in virial equilibrium if its virial parameter is equal to 1 . If the virial parameter is bigger than 1 , turbulence dominates over gravity and the source expands. Otherwise, if the virial parameter becomes smaller than 1 , gravity is the dominating force and the cloud collapses. In reality, additional effects like gas pressure, external pressure and magnetic fields (being ignored in our calculations) influence a cloud's state of equilibrium. Even clouds with $\alpha \gg 1$ can be stable, because these additional terms can change the balance (Bertoldi \& McKee 1992).

In Fig. 9 the correlations between the virial and gas masses are plotted for each model. The value $\alpha=1$ is indicated by a straight black line. For the model of Ossenkopf \& Henning (1994) the majority of the IRDCs have virial parameters slightly above 1 (mean: 6 , median: 2). In contrast, the majority of the sources' virial parameters in the model of Mathis et al. (1977) are slightly below 1 (mean: 2, median 0.7 ). Because the models represent maximum and minimum estimates, the real virial parameters are approximately equal to 1 and the sources, thus, are close to virial equilibrium. This implies that the gas clumps can be stable, but may be at the verge of collapse and star formation.

Our results match those of Dunham et al. (2011), but not quite those of Wienen et al. (2012) and Harju et al. (1993). Wienen et al. (2012) have mean virial parameters of 0.21 for FWHMs smaller than $4 \mathrm{~km} \mathrm{~s}^{-1}$ (and 0.45 for FWHMs larger than $4 \mathrm{~km} \mathrm{~s}^{-1}$, but we do not have any source with such a large line width) by using the Ossenkopf \& Henning model and assuming

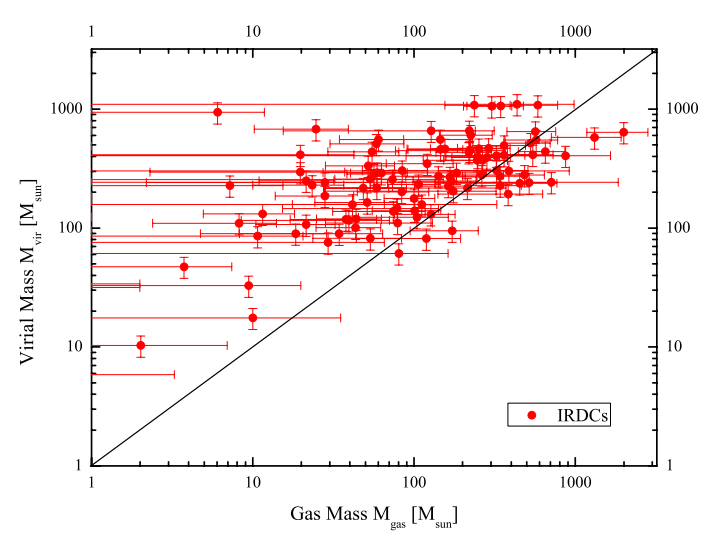

(a) Ossenkopf \& Henning (1994): $\kappa_{\mathrm{OH}}=1.85 \mathrm{~cm}^{2} \mathrm{~g}^{-1}$

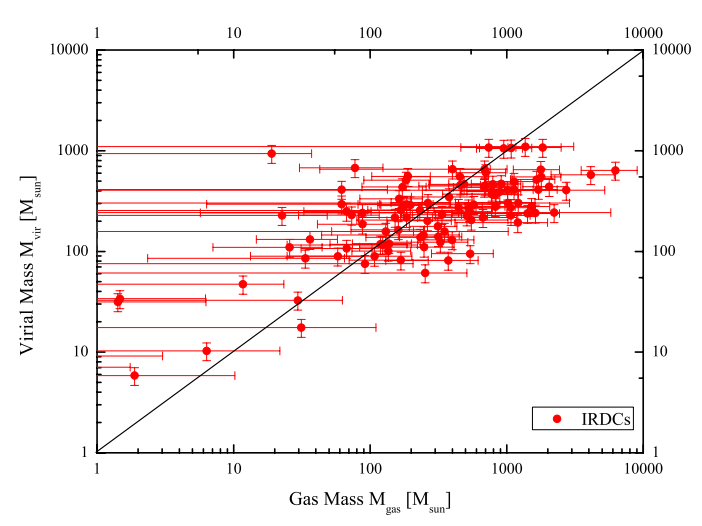

(b) Mathis et al. (1977): $\kappa_{\mathrm{MRN}}=0.6 \mathrm{~cm}^{2} \mathrm{~g}^{-1}$

Fig. 9. Correlation plot between virial and gas mass using the model of Ossenkopf \& Henning (1994) and Mathis et al. (1977). The indicated black straight line represents the line of $\alpha$ being equal to 1 .

the equipartition between magnetic and kinetic energy which reduces their value by a factor 0.5 . Additionally, the difference is, to some degree, certainly due to their lower spectral resolution of $0.7 \mathrm{~km} \mathrm{~s}^{-1}$ compared to our spectral resolution of $0.38 \mathrm{~km} \mathrm{~s}^{-1}$, although Wienen et al. corrected for this in their calculation of virial masses. This reduces their used FWHM, and by that the estimate.

In contrast, the virial parameters of Harju et al. (1993) $\left(=\frac{2 E_{\mathrm{kin}}}{E_{\mathrm{pot}}}\right)$ are between 1.7 and 4 and, therefore, larger than ours. An explanation could be that their cores are more evolved than ours, indicating a more active phase of star formation. Since their sources can be associated with IRAS sources, this is not surprising. This may look like a contradiction to the small line widths (cf. Sect. 4.3). But considering the smaller solid angles and distances, Harju et al. (1991) observed also smaller absolute values of mass, kinetic and potential energies. Since the virial parameter is calculated by the ratio of kinetic and potential energies, the absolute measured order of magnitude does not really matter, but the relation between the energies. And this relation depends on the evolutionary stage of the objects.

\subsubsection{Hydrogen column density and ammonia abundance}

With the ATLASGAL data, we were also able to calculate the column density of molecular hydrogen and the abundance of ammonia compared to hydrogen by using the formula

$N_{\mathrm{H}_{2}}^{19^{\prime \prime}}=\frac{F_{v}^{19^{\prime \prime}} R}{B_{v}\left(T_{\mathrm{D}}\right) \Omega \kappa_{v} \mu m_{\mathrm{H}}}$ 
(Schuller et al. 2009) where $F_{v}^{19^{\prime \prime}}$ is the total flux density measured in dust emission at $870 \mu \mathrm{m}$ within the solid angle of $\Omega=6.66 \times 10^{-9}$ sr $\left(19^{\prime \prime}\right), \mu$ the molecular weight of hydrogen and $m_{\mathrm{H}}$ the weight of one hydrogen atom. For the dust absorption coefficient we adapted $\kappa_{v}=1.85 \mathrm{~cm}^{2} \mathrm{~g}^{-1}$ from Schuller et al. (2009). The column densities of molecular hydrogen in our sample range between $2 \times 10^{20} \mathrm{~cm}^{-2}$ and $3 \times 10^{23} \mathrm{~cm}^{-2}$, with an average of $4 \times 10^{22} \mathrm{~cm}^{-2}$ and a median of $3 \times 10^{22} \mathrm{~cm}^{-2}$ (cf. Table 2). These results match those of Wienen et al. (2012) and Dunham et al. (2011) $\left(\sim 10^{22} \mathrm{~cm}^{-2}\right)$.

Since the ammonia column densities have been calculated with data observed with a $40^{\prime \prime}$ beam, we used the smoothed ATLASGAL data for deriving the ammonia abundances $\chi_{\mathrm{NH}_{3}}=$ $N_{\mathrm{NH}_{3}} / N_{\mathrm{H}_{2}}^{40 \prime} . N_{\mathrm{H}_{2}}^{40 \prime}$ is the hydrogen column density derived with Eq. (4) by inserting the total flux density $F_{v}^{40 "}$ within the solid angle $\Omega=2.95 \times 10^{-8} \mathrm{sr}\left(40^{\prime \prime}\right.$, smoothed data which have been also used for calculating the sample sources' gas masses, cf. Sect. 4.5.2). We find $\mathrm{NH}_{3}$ abundances lie between $6 \times 10^{-9}$ and $5 \times 10^{-7}$ with a median of $2 \times 10^{-8}$. The average abundance is of the order $10^{-8}$ which agrees with the results of Wienen et al. (2012) and Dunham et al. (2011) $\left(\sim 5 \times 10^{-9}-5 \times 10^{-7}\right)$.

\section{Summary and conclusion}

218 candidate IRDCs located between $13^{\circ}$ and $50^{\circ}$ in Galactic longitude and $\pm 1.5^{\circ}$ Galactic latitude have been observed in the ammonia (1,1)- and (2,2)-inversion transitions with the Effelsberg $100 \mathrm{~m}$ radio telescope. In 109 sources we found ammonia (1,1)-inversion lines and discussed the physical conditions within them.

We found that there is a correlation between a source's position within the Galactic plane and the detectability of ammonia. The smaller the absolute value of the source's Galactic latitude, the greater the chance to find ammonia inversion lines. This makes sense, because ammonia is a high density tracer and the gas density is higher in the inner regions of the Galactic plane than in the outer ones. To improve the detection rate, future observations should focus on areas of low Galactic latitudes $(|b|<$ $\left.0.5^{\circ}\right)$. The dust emission maps of the ATLASGAL project confirm this statement, because the majority of compact sources detected by them have been found in this area (Beuther et al. 2012). Vice versa, almost all sources with high submm flux densities have counterparts in the IRDC catalogue (Schuller et al. 2009). Therefore, the detection rate could be increased by building up future IRDC samples on the base of the ATLASGAL data. We checked if our sources can be associated with Spitzer $24 \mu \mathrm{m}$ emission indicating the existence of YSOs embedded within the IRDC candidates. We found the fraction of sample IRDCs having a counterpart in the Spitzer data to be $20.2 \%$, but were not able to find any correlation between the association and the temperatures measured with the ammonia observations.

The observed line widths range between 0.5 and $2.5 \mathrm{~km} \mathrm{~s}^{-1}$, some up to $4 \mathrm{~km} \mathrm{~s}^{-1}$, and are much higher than expected for purely thermal broadening $\left(\approx 0.2 \mathrm{~km} \mathrm{~s}^{-1}\right)$. Therefore, turbulence has to play an important role to stabilise IRDCs. Additionally, the FWHMs are significantly smaller than the ones of HMPOs, but higher than values measured in low-mass cores. We interpret this as additional evidence for IRDCs representing very early stages of high-mass star formation, although not all of our sample IRDCs will be able to actually form high-mass stars. The rotation temperatures $\left(8 \leq T_{\text {rot }} / \mathrm{K} \leq 30\right)$ are on average $15 \mathrm{~K}$. Thus, IRDCs are cooler than HMPOs $(\sim 22 \mathrm{~K})$. The ammonia column densities of IRDCs are about an order of magnitude smaller than the ammonia column densities of HMPOs.

Using ATLASGAL data, we computed the gas masses, column densities of molecular hydrogen and ammonia abundance relative to molecular hydrogen. We found molecular hydrogen column densities on the order of $10^{22} \mathrm{~cm}^{-2}$ on average implying an average ammonia abundance on the order of $10^{-8}$.

The virial masses derived for the cores within the IRDCs are between 100 and a few $1000 M_{\odot}$. The corresponding virial parameters are on the order of $\sim 1$. Thus, the majority of observed IRDCs is close to virial equilibrium and hence consists of potential candidates for pre-protostellar regions of future star formation.

Acknowledgements. This research has made use of the catalogue of Infrared Dark Clouds and Cores based on MSX data and was built up by Simon et al. as well as the dust emission maps of the ATLASGAL project. L.B. acknowledges support from CONICYT projects FONDAP 15010003 and Basal PFB-06.

\section{References}

Bergin, E. A., \& Tafalla, M. 2007, ARA\&A, 45, 339

Bertoldi, F., \& McKee, C. F. 1992, ApJ, 395, 140

Beuther, H., Churchwell, E. B., McKee, C. F., \& Tan, J. C. 2007, Protostars and Planets V, 165

Beuther, H., Tackenberg, J., Linz, H., et al. 2012, ApJ, 747, 43

Buisson, G., Desbats, L., Duvert, G., et al. 2007, GILDAS GreG Documentation Churchwell, E., Walmsley, C. M., \& Cesaroni, R. 1990, A\&AS, 83, 119

Danby, G., Flower, D. R., Valiron, P., Schilke, P., \& Walmsley, C. M. 1988, MNRAS, 235, 229

Dunham, M. K., Rosolowsky, E., Evans, II, N. J., Cyganowski, C., \& Urquhart, J. S. 2011, ApJ, 741, 110

Egan, M. P., Shipman, R. F., Price, S. D., et al. 1998, ApJ, 494, L199

Harju, J., Walmsley, C. M., \& Wouterloot, J. G. A. 1991, A\&A, 245, 643

Harju, J., Walmsley, C. M., \& Wouterloot, J. G. A. 1993, A\&AS, 98, 51

Hily-Blant, P., Pety, J., Guilloteau, S., et al. 2006, GILDAS CLASS Documentation, 1.1 edn.

Ho, P. T. P., \& Townes, C. H. 1983, ARA\&A, 21, 239

Kunth, D., Guiderdoni, B., Heydari-Malayeri, M., \& Thuan, T. X. 1996, The interplay between massive star formation, the ISM and galaxy evolution

MacLaren, I., Richardson, K. M., \& Wolfendale, A. W. 1988, ApJ, 333, 821

Mathis, J. S., Rumpl, W., \& Nordsieck, K. H. 1977, ApJ, 217, 425

Molinari, S., Brand, J., Cesaroni, R., \& Palla, F. 1996, A\&A, 308, 573

Ossenkopf, V., \& Henning, T. 1994, A\&A, 291, 943

Perault, M., Omont, A., Simon, G., et al. 1996, A\&A, 315, L165

Peretto, N., \& Fuller, G. A. 2009, A\&A, 505, 405

Peretto, N., Fuller, G. A., Plume, R., et al. 2010, A\&A, 518, L98

Pillai, T., Wyrowski, F., Carey, S. J., \& Menten, K. M. 2006, A\&A, 450, 569

Ragan, S. E., Bergin, E. A., \& Wilner, D. 2011, ApJ, 736, 163

Reid, M. J., Menten, K. M., Zheng, X. W., et al. 2009, ApJ, 700, 137

Rosolowsky, E., Dunham, M. K., Ginsburg, A., et al. 2010, ApJS, 188, 123

Schilke, P. 1989, Master's Thesis

Schreyer, K., Henning, T., Koempe, C., \& Harjunpaeae, P. 1996, A\&A, 306, 267

Schuller, F., Menten, K. M., Contreras, Y., et al. 2009, A\&A, 504, 415

Simon, R., Jackson, J. M., Rathborne, J. M., \& Chambers, E. T. 2006, ApJ, 639, 227

Sridharan, T. K., Beuther, H., Schilke, P., Menten, K. M., \& Wyrowski, F. 2002, ApJ, 566, 931

Sridharan, T. K., Beuther, H., Saito, M., Wyrowski, F., \& Schilke, P. 2005, ApJ, 634, L57

Tafalla, M., Myers, P. C., Caselli, P., \& Walmsley, C. M. 2004, A\&A, 416, 191

Walmsley, C. M., \& Ungerechts, H. 1983, A\&A, 122, 164

Wienen, M., Wyrowski, F., Schuller, F., et al. 2012, A\&A, 544, A146

Wilcock, L. A., Ward-Thompson, D., Kirk, J. M., et al. 2012, MNRAS, 424, 716

Pages 9 to 12 are available in the electronic edition of the journal at http://www . aanda.org 


\section{Appendix A: Derivation of used formulas}

\section{A.1. Rotation and kinetic temperature}

As described in Sect. 3, the hyperfine fit routine returns the best fit parameters of the brightness temperature, $T_{\mathrm{mb}}$, optical depth, $\tau$, velocity of rest, $V_{\mathrm{lsr}}$, and line width FWHM, $\Delta v$, (with errors).

The brightness temperature $T_{\mathrm{mb}}$ describes the brightness temperature of a source and depends on the beam this source is observed with by returning the mean value over this beam. It is needed to calculate the excitation temperature.

The excitation temperature $T_{\text {ex }}$ is no physical temperature, but describes the ratio between two population levels $u(\mathrm{p})$ and $l$ (ow) via

$\frac{n_{\mathrm{u}}}{n_{\mathrm{l}}}=\frac{g_{\mathrm{u}}}{g_{\mathrm{l}}} \exp \left(-\frac{\Delta E}{k T_{\mathrm{ex}}}\right)$

where $n_{\mathrm{i}}$ is the numbers of particles in the state $\mathrm{i}$ and $g_{\mathrm{i}}$ its statistical weight. $\Delta E$ represents the energy difference between the states and $k$ the Boltzmann constant. For the IRDCs temperatures above $10 \mathrm{~K}$ are expected. Thus, (by applying the Rayleigh-Jeans law) the brightness and excitation temperatures are simplified connected by

$T_{\mathrm{ex}}=\frac{T_{\mathrm{mb}}}{\eta_{\mathrm{f}}\left(1-\mathrm{e}^{-\tau}\right)}+T_{\mathrm{BG}}$

with $\tau$ being the source's optical depth and $T_{\mathrm{BG}}$ the background temperature (being about $2.7 \mathrm{~K}$ ). $\eta_{\mathrm{f}}$ represents the beam-filling factor. It describes the fraction of the antenna pattern being received from the source. In the case of extended and not clumpy sources, the beam-filling factor is equal to 1 . We assume this case for the sample. The excitation temperatures are calculated for both transition lines.

The rotation temperature $T_{\text {rot }}$ is defined similar to the excitation temperature by

$\frac{n_{\mathrm{i}}^{1}}{n_{\mathrm{j}}^{1}}=\frac{g_{\mathrm{i}}}{g_{\mathrm{j}}} \exp \left(-\frac{\Delta E}{k T_{\mathrm{rot}}^{\mathrm{ij}}}\right)$.

But in contrast to the excitation temperature, the rotation temperature does not describes the ratio between different levels being split by inversion, but between the levels of quantum numbers $J$ and $K$ (total angular momentum and its absolute projection along the $z$-axis). We are interested in the rotation temperatures of the metastable inversion levels with $J=K$ (non-metastable inversion levels: $J>K$ ). These levels cannot be populated or depopulated by radiation. Therefore, their population numbers are high enough to emit measurable line intensities. Furthermore, metastable levels interact only via collisions (Ho \& Townes 1983; Schilke 1989) and are useful for the calculation of gas temperatures.

The remaining problem is that we do not know the exact population numbers and have to approximate them by the column densities. For lines being observed in the same region the ratio of the population numbers should be equal to the ratio between the corresponding column densities depending on the ratio of the lines' optical depths:

$\frac{\tau^{J K}}{\tau^{J^{\prime} K^{\prime}}} \approx \frac{K^{2}}{J(J+1)} \frac{J^{\prime}\left(J^{\prime}+1\right)}{K^{\prime 2}} \frac{2 J^{\prime}+1}{2 J+1} \frac{\Delta v_{1}}{\Delta v_{2}} \frac{N_{\mathrm{l}}(J, K)}{N_{\mathrm{l}}\left(J^{\prime}, K^{\prime}\right)} \frac{v_{J K}}{v_{J^{\prime} K^{\prime}}} \frac{T_{\mathrm{ex}}^{J^{\prime} K^{\prime}}}{T_{\mathrm{ex}}^{J K}}$

where $\tau^{J K}$ is the optical depth of the $(J, K)$-inversion line, $\Delta v_{J}$ its line width FWHM, $N_{\mathrm{l}}(J, K)$ its column density and $T_{\mathrm{ex}}^{J K}$ its excitation temperature. Because only the (1,1)- and (2,2)-inversion lines have been studied, it is $J=K=2$ and $J^{\prime}=K^{\prime}=1$ in the following calculations.

Inserting this into Eq. (A.3), one gets:

$T_{\text {rot }}=-\frac{E}{x \frac{\tau^{J K}}{\tau^{J^{\prime} K^{\prime}}}}$

where $E=\frac{h v_{i j}}{k}=41.5 \mathrm{~K}$ (Ho \& Townes 1983) and

$x=\frac{K^{\prime 2}}{J^{\prime}\left(J^{\prime}+1\right)} \frac{J(J+1)}{K^{2}} \frac{2 J^{\prime}+1}{2 J+1} \frac{\Delta v_{2}}{\Delta v_{1}} \frac{v_{J^{\prime} K^{\prime}}}{v_{J K}} \frac{T_{\mathrm{ex}}^{J K}}{T_{\mathrm{ex}}^{J^{\prime} K^{\prime}}}$.

Following the instruction of Schilke (1989) and Ho \& Townes (1983), there are four cases one has to consider for calculating the rotation temperature. These cases differ in the optical depth of each inversion line:

1. both inversion lines are optical thick:

$$
T_{\text {rot }}=-\frac{E}{\ln \left(x \frac{f_{1}}{f_{2}} \frac{\tau^{22}}{\tau^{11}}\right)}
$$

where $\tau^{\mathrm{ii}}$ is the optical depth of the (i, i)-inversion line and $f_{i}$ is the relative intensity of the main hyperfine component. For the given transitions, there is $f_{1}=0.5$ and $f_{2}=0.796$ (Ho \& Townes 1983).

2 . only the $(1,1)$-inversion line is optical thick and the $(2,2)$ inversion line is optical thin:

$$
T_{\text {rot }}=-\frac{E}{\ln \left[-\mathrm{x} \frac{f_{1}}{\tau^{11} f_{2}} \ln \left(1-\frac{\tau^{22}}{\tau^{11}}\left(1-\mathrm{e}^{-\tau^{11}}\right)\right)\right]}
$$

3. both inversion lines are optical thin:

$$
T_{\mathrm{rot}}=-\frac{E}{\ln \left(-x \frac{f_{1}}{f_{2}} \frac{T_{\mathrm{mb}}^{1}}{T_{\mathrm{mb}}^{2}}\right)}
$$

where $T_{\mathrm{mb}}^{\mathrm{i}}$ represents the brightness temperature of the (i, i)inversion line.

4. only the (1,1)-inversion line is detected, the $(2,2)$-inversion line is not:

$$
T_{\text {rot }}=\frac{-E}{\ln \left[-\frac{0.282}{\tau^{11}} \ln \left(1-\frac{T_{\mathrm{mb}}^{2}}{T_{\mathrm{mb}}}\left(1-\mathrm{e}^{-\tau^{11}}\right)\right)\right]} .
$$

In this case, the (2,2)-inversion line lies within the noise. To be able to continue with the calculations, $T_{\mathrm{mb}}^{2}$ has been given the triple value of the average root-mean-square noise $(\mathrm{rms}=0.0925 \mathrm{~K})$. It is important to emphasise that in this case it is not possible to derive exact rotation temperatures, but only upper limit estimations!

Having the rotation temperature of ammonia, we were able to calculate the kinetic temperature of a source's gas by using the approximation of Tafalla et al. (2004):

$T_{\text {kin }}=\frac{T_{\text {rot }}}{1-\frac{T_{\text {rot }}}{E} \ln \left[1+1.1 \exp \left(-\frac{15.7 \mathrm{~K}}{T_{\text {rot }}}\right)\right]}$.

This approximation has been derived with Monte Carlo models and gives an accuracy of 5\% in the range between 5 and $20 \mathrm{~K}$. Because the majority of the sample IRDCs are within this range, their errors are set to this. 


\section{A.2. Column density}

As mentioned before in Sect. A.1, the column density is related to a source's optical depth (Schilke 1989). If one neglects the background radiation and expresses the excitation temperature in terms of the optical depth, following Schilke (1989), the column density of the $(1,1)$-inversion level $N^{11}$ can by calculated by

$N^{11} \approx 2 T_{\mathrm{mb}} \frac{\tau}{1-\mathrm{e}^{-f_{1} \tau^{11}}} \frac{3 h}{8 \pi^{3}} \frac{\sqrt{\pi}}{2 \sqrt{\ln (2)}} \frac{J^{\prime}\left(J^{\prime}+1\right)}{K^{\prime 2}} \frac{\Delta v_{1}}{\mu^{2}} \frac{k}{h v_{1}}$

where $\mu=1.476$ Debye is the electric dipole moment and $v_{1}=23694.496 \mathrm{MHz}$ the laboratory frequency of the $\mathrm{NH}_{3}$ $(1,1)$-inversion transition. To derive the total column density,
$N_{\mathrm{NH}_{3}}$, we assumed that the sources are in thermal equilibrium. In this case, the column density follows a Boltzmann distribution. Additionally, we took into account that Eq. (A.12) calculates the column density of para- $\mathrm{NH}_{3}$ being the ammonia inversion levels with $K \neq 3 n$ ( $n$ being an integer) where the hydrogen spins are not parallel. In contrast, the states with parallel hydrogen spins are called ortho- $\mathrm{NH}_{3}$ (with $K=3 n$ ). The statistical weight $g_{J K}$ of ortho- $\mathrm{NH}_{3}$ is twice the one of para- $\mathrm{NH}_{3}$. Therefore, for calculating the total column density of ammonia, one has to take the triple of column density of para- $\mathrm{NH}_{3}$ (cf. Schilke 1989):

$N_{\mathrm{NH}_{3}}=\frac{3 N^{11}}{2\left(2 J^{\prime}+1\right)} \sum_{1,1} g_{11} \exp \left[\left(23.4 \mathrm{~K}-\frac{h v_{1}}{k}\right) \frac{1}{T_{\text {rot }}}\right]$. 
R.-A. Chira et al.: Characterization of infrared dark clouds

\section{Appendix B: Position of sample IRDCs within the Galactic plane}

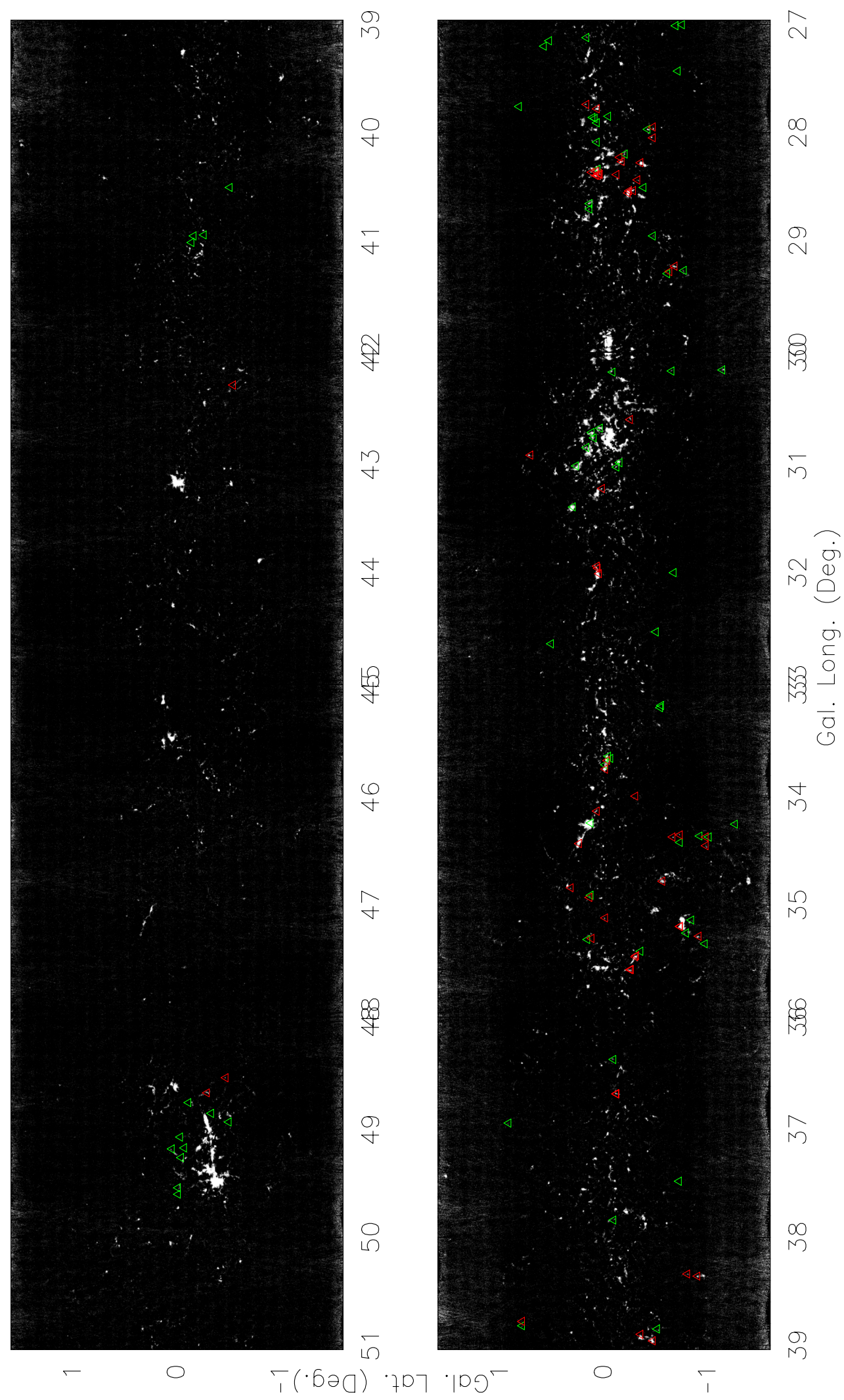

Fig. B.1. ATLASGAL dust emission map with indicated sample IRDCs in the range of $1=27^{\circ}$ to $51^{\circ}$ (Schuller et al. 2009). The red triangles indicate the IRDCs with detected ammonia lines, the green triangles the ones without detected ammonia lines. 
A\&A 552, A40 (2013)

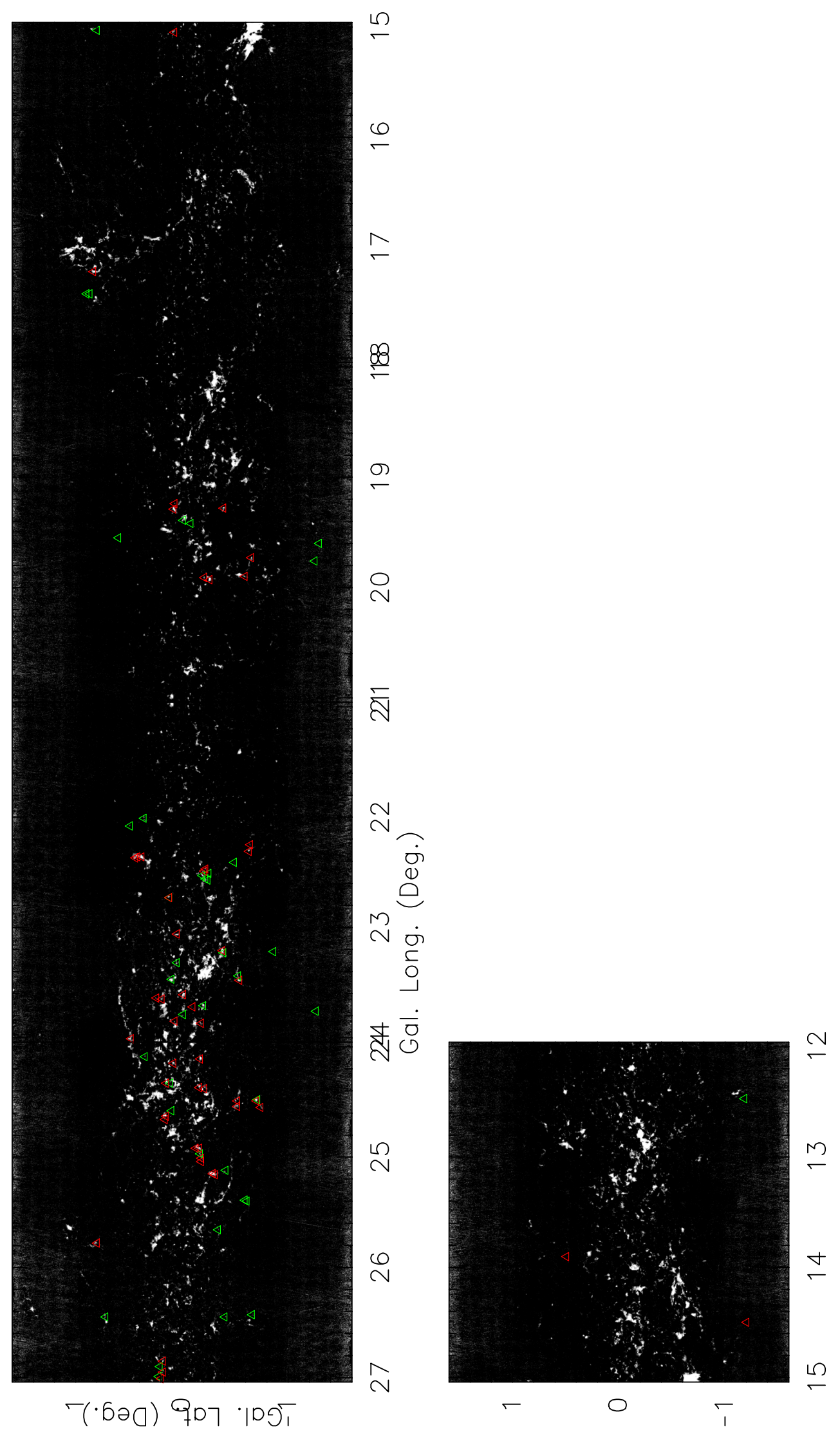

Fig. B.2. ATLASGAL dust emission map with indicated sample IRDCs in the range of $1=12^{\circ}$ to $27^{\circ}$ (Schuller et al. 2009). The red triangles indicate the IRDCs with detected ammonia lines, the green triangles the ones without detected ammonia lines. 